\title{
Latent inhibition of the GSR conditioned to words
}

\author{
IRVING MALTZMAN, DAVID C. RASKIN, and CRAIG WOLFF \\ University of California, Los Angeles, Los Angeles, California 90024
}

\begin{abstract}
The GSR measure of the orienting reflex was obtained from 90 college students in a semantic conditioning and generalization experiment. Different groups received 0,20 , or 40 presentations of different words other than the CS word prior to the start of conditioning. Latent inhibition was manifested in differences in the CR peak attained by the three groups and the number of trials required to attain the peak. They also differed significantly in the magnitude of their responses to UCS omission during extinction. The three groups did not differ in the magnitude of their GSR to control words. It was concluded that conditioned attention theory as presently formulated cannot adequately account for all of these results. An alternative interpretation was offered in terms of a theory which asserts that classical conditioning of the GSR involves an active problem solving discovery process that leads to the generation of a voluntary OR. It is manifested in the form of a GSR-CR. Preconditioning habituation sessions serve as a masking task which interferes with successful problem solving, discovering the signal for the UCS.
\end{abstract}

The principal purpose of this experiment was to investigate the effects of different amounts of preconditioning habituation to different words on subsequent conditioning of the GSR to a specific word. Soviet investigators, beginning with Pavlov (1927), have asserted that the elaboration of conditioned reflexes is facilitated by the prior occurrence of an optimal degree of orienting to the CS. If the CS induces orienting reflexes (ORs) that are too weak or too strong, conditioning will be retarded or may not occur at all. An obvious experimental implication of the above assertion is that prior habituation of the OR induced by the CS will make acquisition of the CR more difficult. According to Soviet investigators (Voronin, Leontiev, Luria, Sokolov, \& Vinogradova, 1965), because of the nonoptimal degree of attention produced by habituation of the OR, the level of cortical excitability necessary for formation of a temporary connection would be less than optimal.

The technique of habituating the OR prior to conditioning has theoretical value because it permits the manipulation of the OR and its measures independently of the measures of the CR, provided that the $C R$ is of a different form than the OR which

Portions of the results reported here were presented at the 76th Annual Meeting of the American Psychological Association, San Francisco, September 1968. This study was made possible in part by funds provided by the Carnegie Corporation of New York and by United States Public Health Service Research Grant MH4684 from the National Institute of Mental Health. For the analyses of the results of the experiment reported here, computing assistance was obtained from the Health Sciences Computing Facility, UCLA, sponsored by NIH Grant FR-3. David C. Raskin is now at the University of Utah. Requests for reprints should be sent to Irving Maltzman, Department of 'Psychology, University of California, Los Angeles, California 90024. is concurrently recorded. Studies conducted in the Soviet Union (Sokolov, 1963; Voronin et al., 1965) are described in insufficient detail to adequately evaluate the evidence in support of this proposition, and most such studies employed the now generally discredited Ivanov-Smolensky technique of conditioning with verbal reinforcement (Dmitriev, 1962).

Independent of the Soviet work, the corresponding research problem was introduced in the West and its effects labeled "latent inhibition" by Lubow and his associates (Lubow \& Moore, 1959). The phenomenon in question refers to the decrement in CRs following nonreinforced preexposure of the stimulus subsequently serving as the CS. There have now been a large number of studies of latent inhibition, many by Lubow and his associates as well as other investigators, who have employed human and infrahuman subjects in a variety of different kinds of experimental situations (Lubow, 1973a, 1973b). The great range of subjects, response measures, and different experimental arrangements that have been employed in positive demonstrations of latent inhibition indicate that it is a phenomenon of considerable generality and reliability (e.g., Carlton \& Vogel, 1967; Lubow, 1973a, 1973b; Lubow, Alek, \& Arzy, 1975; Lubow, Rifkin, \& Alek, 1976; Lubow, Schnur, \& Rifkin, 1976). Studies of latent inhibition in GSR conditioning, however, have yielded mixed results. Zeiner (1970) apparently found no latent inhibition effect in a discriminative conditioning situation. Surprisingly enough, Silver (1973) purported to show superior conditioning for his latent inhibition groups, which received 16 preexposures of the CS as compared to groups receiving 1 and 4 preexposures. In contrast, Surwit and Poser (1974) showed a latent inhibition 
effect in groups preexposed to the CS as compared to groups preexposed to an irrelevant stimulus. Clearly, more research is needed in order to adequately determine the variables responsible for the obtained effects in GSR conditioning and to provide an adequate theoretical account for these effects.

After reviewing a number of different theoretical interpretations of latent inhibition, including habituation of the OR, Lubow (1973a) rejected these interpretations, concluding that a viable interpretation of latent inhibition must include learning and attention as theoretical processes. This conclusion has been formulated as conditioned attention theory (Lubow, Alek, \& Arzy, 1975; Lubow, Rifkin, \& Alek, 1976; Lubow, Schnur, \& Rifkin, 1976).

Conditioned attention theory treats attention as a hypothetical construct with the characteristics of a classically conditioned response. The attention response is conditionable, but in contrast to the usual $\mathrm{CR}$, it is evoked by the CS on its first presentation, prior to the occurrence and possibility of reinforcement by the UCS. The effect of the UCS is to maintain the attention response induced by the CS or prevent its habituation. The absence of a second significant event following the CS results in a relatively permanent decrease in the associability of that CS. Thus there is a concomitant learning process which is conceived of as the conditioning of inattention. Conditioned attention theory has much in common with proposals by MacKintosh (1975), and implies a two-stage theory of conditioning. But not only does this interpretation have much in common with interpretations proposed by MacKintosh, it is strikingly similar to the OR as formulated by Sokolov (1963) and others (Maltzman, 1977; Pendery \& Maltzman, 1977). There are, however, certain important differences, particularly in relation to GSR conditioning in normal adult humans.

Our interpretation is that the usual GSR conditioning in normal adults is a form of conditioning of the OR, a form of conditioned attention (Maltzman, 1977; Pendery \& Maltzman, 1977). However, there are different forms of the OR. One is an involuntary OR that is primarily a consequence of stimulus change, novelty, and the collative variables emphasized by Berlyne (1960). A second and related form, voluntary $\mathrm{OR}$, is a consequence of the discovery of the significance of the CS, the discovery that it is a signal for the UCS. Discovered significance of the CS results in a large OR that is taken to be the CR to this stimulus. It is maintained by the UCS for varying numbers of trials. With continued conditioning trials, the CR-OR habituates despite continued reinforcement. This is simply another manifestation of the characteristic of the OR. It habituates with uniformity in stimulating conditions (Sokolov, 1963).

Given the above analysis of classical conditioning of the GSR, the question in relation to latent inhibition of the GSR is whether preconditioning expo- sures of the CS affect the problem solving strategies of the participants, the magnitude of the OR or attention response, or both.

There were three groups of college students in the present experiment. Group 40 received 40 different neutral words prior to conditioning; Group 20 received the first 20 words presented to Group 40; Group 0 received the CS word without the prior presentation of any words. It should be noted that this procedure differs from past latent inhibition studies in that the specific CS employed in conditioning was not preexposed. Different stimuli of the same general class, different words, were preexposed. An interesting theoretical question, then, is whether there will be a latent inhibition effect under such conditions, whether there will be a decrement specific to the CS word, or whether the decrement in GSR will occur to all words, control filler words as well as the CS.

Conditioned attention theory is ambiguous on this point. Since the specific CS word was not preexposed, conditioned inattention should not occur to the CS word and therefore there should be no latent inhibition in the CR induced by that word. Or, it might be argued from conditiond attention theory, since habituation occurs to all words as the result of repeated presentations of different words during the preconditioning habituation session, there is generalization of the inhibitory effect. Overall responsivity should therefore be depressed during conditioning. There would be a decrement in responsivity to all words, control as well as CS words, but differential conditioning should not be affected. Either implication drawn from conditioned attention theory suggests that a latent inhibition effect should not occur. Differential conditioning of the GSR to words should not be affected as a result of preexposure of words unrelated to the CS word (e.g., Carlton \& Vogel, 1967; Siegel, 1969).

Cephalic vasomotor activity was recorded as well as the GSR during the course of the experiment. We had hoped thereby to examine the effects of different amounts of preconditioning habituation of the OR indexed by cephalic vasodilation on the acquisition of a conditioned defensive reflex indexed by cephalic vasoconstriction (Sokolov, 1963) as well as the GSR measure of the OR. Since we failed to obtain reliable evidence of cephalic vasomotor conditioning in this as well as other experiments performed in this laboratory (Maltzman, Langdon, Pendery, \& Wolff, 1977; Maltzman, Weissbluth, \& Wolff, 1978), we will not discuss these vasomotor measures further.

A final aim of this study was to vary the number of conditioning trials prior to the presentation of a single semantic generalization test trial, since previous experiments with a smaller number of conditioning trials failed to show evidence of semantic generalization when a single test trial was employed (Maltzman, Gould, Barnett, Raskin, \& Wolff, 1977). 
Different subgroups in the present experiment therefore received a semantic generalization test trial after $5,15,25,35$, or 45 conditioning trials.

\section{METHOD}

\section{Subjects}

There were 90 University of California, Los Angeles introductory psychology students who participated in the experiment for partial fulfillment of a course requirement. Half the participants were men and half were women.

\section{Procedure.}

An equal number of men and women were assigned to each of three habituation groups. Group 40 received $2 \mathrm{~min}$ of silence followed by 40 different words prior to the start of conditioning. Group 20 received $6 \mathrm{~min}$ of silence followed by 20 habituation words which were the first 20 words presented Group 40. Group 0 waited in silence for 10 min prior to the start of conditioning, the presentation of the first CS word.

Each habituation group was subdivided into five generalization subgroups which received a single generalization test word, STEM, after $5,15,25,35$, or 45 conditioning trials. This generalization test word simply appeared as one of the filler words interspersed between conditioning trials.

Conditioning consisted of 45 presentations of the word PLANT followed in $10 \mathrm{sec}$ by a .5-sec white noise at $95 \mathrm{~dB}$ (A). From two to four unrelated neutral filler words were interspersed among the conditioning trials. The interword interval and the time between the UCS and a subsequent filler word varied between 10 and $16 \mathrm{sec}$. Following the 45 conditioning trials without interruption, all participants received 10 extinction trials which consisted of the presentation of the CS word PLANT without the UCS. As was the case during conditioning, extinction trials were interspersed among neutral filler words.

After the experiment was concluded, the participants were administered a questionnaire designed to obtain a measure of the extent to which they verbalized the relevant stimulus contingencies. They rated the critical and selected filler words on a semantic differential, and were administered the short form of the Taylor Manifest Anxiety Scale.

\section{Apparatus.}

Palmar GSRs were recorded using two 20-mm circular disk electrodes fitted in 5-mm-deep plastic cups. The cups contained electrode paste made from agar and a $1 \%$ zinc sulfate solution. The GSR electrodes were held in place by plastic pressure clips. With a $40-\mu \mathrm{A}$ current, palmar resistance was continuously monitored via a Darrow-type bridge on one channel of a dual-channel dc Sanborn 320 amplifier-recorder. The vasomotor response mea sure of blood volume was recorded by means of a Starling Corporation photoplethysmograph with the light-photocell face of the transducer in contact with the skin on the left temporal area of the head. It was maintained in place by an adjustable headband.

The GSR was scored as the largest resistance change occurring within the interval . $5-5 \mathrm{sec}$ following the CS word "plant," the UCS, the test word "STEM," and the different neutral filler words immediately preceding, $C_{1}$, and following, $C_{2}$, each presentation of a conditioning, generalization test, and extinction trial. Responses to the words presented Groups 20 and 40 during preconditioning habituation were also scored. Second-interval or anticipatory responses (Stern, 1972) occurring immediately prior to the UCS during conditioning were not scored because we have found that these responses add little to the information obtained from first-interval responses (Maltzman, Weissbluth, \& Wolff, 1978). Third-interval responses were scored during extinction for all groups. These responses to omission of the UCS were scored in the interval $.5-5 \mathrm{sec}$ following the interval where the UCS had occurred during the conditioning phase.

Log conductance base level at the time each stimulus was presented was also determined. Prior to statistical analyses, the GSR was subjected to a log conductance change transformation. The .05 confidence level for a two-tailed test was employed in all statistical analyses.

\section{RESULTS}

Distributions of the magnitude of the GSR and log conductance level to the first word were determined for men and for women separately within each of the habituation groups. The students above the median for a given response distribution were designated as high-OR students, while those below the median were designated as low-OR students.

Analyses of variance of the GSR and log conductance level following the presentation of the first word indicated no reliable differences as a consequence of sex or habituation groups. The latter result indicates that simply waiting varying amounts of time prior to the presentation of the first word did not differentially affect the magnitude of the initial phasic or tonic OR.

As to be expected, presentation of different words during the preconditioning habituation phase resulted in a decrement in magnitude of the GSRs evoked by these words. In Group 20, the Trials effect was $\mathrm{F}(19,456)=18.92, \mathrm{MS}_{\mathrm{W}}=.295$, while in Group 40 the Trials effect was $F(39,936)=8.55$, $\mathrm{MS}_{\mathrm{W}}=.289$.

\section{Conditioning.}

Figure 1 offers a comparison of the mean GSR magnitudes induced by the CS word in the 0-, 20-, and 40-trial preconditioning habituation groups. Responses to the $C_{1}$ and $C_{2}$ control words have been omitted for the sake of clarity. The relatively rapid rise of the CR to different peaks for the three groups as well as its decrease despite reinforcement on every trial is strikingly apparent. The mean GSR

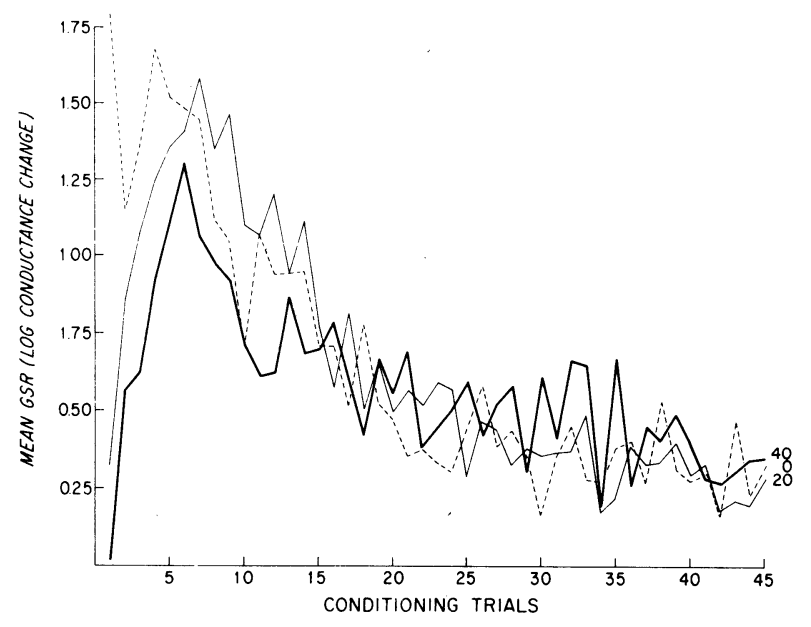

Figure 1. Mean magnitude of the galvanic skin response (GSR) evoked by the conditioned stimulus (CS) in groups receiving 0 , 20, or $\mathbf{4 0}$ different words prior to conditioning. Mean galvanic skin responses (GSRs) to control words have been omitted for the sake of clarity. 


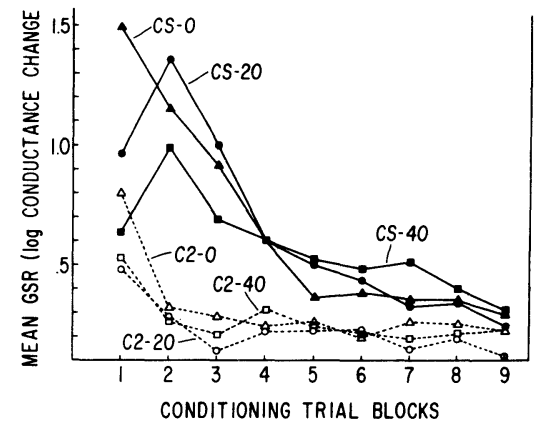

Figure 2. Mean magnitude of the galvanic skin responses (GSRs) evoked by the conditioned stimulus (CS) and the following control words, $C_{2}$, averaged over blocks of five trials in groups receiving 0,20 , or 40 different words prior to conditioning.

magnitude induced by the CS and $\mathrm{C}_{2}$ control words averaged over blocks of five trials each may be seen in Figure 2.

It shows that, while habituation clearly occurred in the responses to the control words as well as to the CS, it is evident that there is still differentiation between the responses to the critical and noncritical stimuli despite the low level of responsivity present at the end of the conditioning phase.

Decrements in the magnitude of the CR or inhibition of reinforcement (Hull, 1943) is characteristic of the GSR (Kimmel \& Burns, 1975; Maltzman, Weissbluth, \& Wolff, 1978). We would ascribe it to the nature of the GSR-CR as a manifestation of an OR. Uniformity in the stimulating conditions of the experiment permits the formation of a neuronal model or the correct anticipations of the events that will occur. The CR-OR habituates as a consequence (Sokolov, 1963). We assume that inhibition of reinforcement of skeletal and glandular CRs (Pavlov, 1927) is largely an indirect reflection of the habituation of the OR and the concomitant lowered level of arousal.

The effectiveness of preconditioning habituation is readily evident in an examination of the mean GSR magnitude to the first CS that may be seen in Figure 1. This response, of course, occurred prior to the first presentation of the UCS. Mean GSR magnitude to the initial CS for Groups 0, 20, and 40 were 1.79 , .32 , and .02 , respectively, indicating the effect of the varying number of words administered prior to the start of conditioning.

Further examination of Figure 1 indicates that, except for Group 0, the curves of the CR showed a progressive increase from the first trial to no more than the eighth trial, and then a gradual decrease. Group 0 reached a peak CR of 1.67 to the fourth CS; Group 20 reached its peak CR of 1.58 to the seventh CS, and Group 40 reached its peak CR of 1.30 to the sixth CS. Individual comparisons by $U$ tests indicated that the $C R$ peak obtained by Group 0 was significantly greater than for Group 40 $(z=4.02)$. No other differences were significant.
Thus, reliable evidence of latent inhibition appears to have been obtained.

It is interesting to note that, in Group 0, the GSR to the first CS reflecting an OR to a novel stimulus resulted in the largest response to a CS found in the three groups. But the conditioning curve for Group 0 did not continue to increase from that initial response level. The response to the second CS was greatly reduced in magnitude, then increased from this new level to its maximum before gradually declining, as did the other groups. This decrease in the magnitude of the GSR following the initial CS and its subsequent increase suggests that conditioning of the GSR is not simply dishabituation of a previously habituated $O R$, as has been claimed (Stewart, Stern, Winokur, \& Freedman, 1961). Likewise, the conditioned GSR does not appear to be either the maintenance through sensitization or increased drive of an involuntary OR induced by the stimulus change or the maintenance of attention. We assume that the initial GSR in Group 0 reflects an involuntary $O R$ to a novel stimulus while the subsequent growth of the GSR-CR to a new asymptote reflects a voluntary OR generated by the students' successful problem-solving activity (Maltzman, 1977; Pendery \& Maltzman, 1977).

In order to avoid the complexities introduced by the OR to the novel first CS in Group 0, and because this group did not have a $C_{1}$ control word on the first trial, analyses of variance and orthogonal polynomials were conducted on the last 44 conditioning trials. Results of the analyses are essentially what might be expected from examination of the figures. Differential semantic conditioning occurred, with Words showing a significant main effect, $\left[\mathrm{F}(2,144)=85.45, \mathrm{MS}_{\mathrm{W} 2}=1.824\right]$.

There was also a significant Trials effect $[F(43,3096)$ $\left.=21.27, \mathrm{MS}_{\mathrm{W} 1}=.371\right]$ and a significant Words by Trials interaction $\left[\mathrm{F}(86,6192)=6.80, \mathrm{MS}_{3}=\right.$ .299]. The orthogonal polynomials analysis also indicated a significant interaction in the linear components of the response trends to the control and CS words $\left[\mathrm{F}(2,144)=59.36, \mathrm{MS}_{\mathrm{W}}=.867\right]$. The decline in response to the CS word was disproportionately greater than the decline to the control words over trials. The linear components of the response trends to all words were negative in sign. A significant $O R$ by Words interaction $\left[\mathrm{F}(2,144)=4.56, \mathrm{MS}_{\mathrm{W} 2}=\right.$ $1.824]$ indicated that the high-OR participants showed greater differential semantic conditioning than low-OR participants. Amount of preconditioning habituation was not a main effect, appearing only as an interaction with Trials and as a difference in the linear components of the response trends in the three groups to the control and critical CS words. Examination of the linear components in the significant Words by Habituation interaction $\left[\mathrm{F}(4,144)=4.13, \mathrm{MS}_{\mathrm{W}}=\right.$ .867] indicated that the disproportionately greater decline in responsivity to the $C_{1}$ control words in 
Group 0 than in Groups 20 and 40 was primarily responsible for the interaction. Another contributing factor was the greater difference between CS and control words in Groups 0 and 20 than in Group 40.

A reasonable interpretation of the absence of a main effect due to latent inhibition is that all groups showed a marked decline, habituation, during conditioning regardless of the degree of preconditioning habituation. This left little room for further effects of latent inhibition to manifest itself. A "floor" effect prevented its appearance as a main effect. Another, and not necessarily incompatible, interpretation is that the effects of habituation prior to conditioning are short lived, at least given the number of preconditioning habituation trials employed in the present experiment. Latent inhibition effects that we shall report in connection with responses to UCS omission, however, are contrary to the latter hypothesis.

The 44 conditioning trials were reanalyzed with between-subject levels constituted by a new categorization of the participants. The students scoring above and below the median basal conductance level at the first word in habitutation or the first CS for Group 0 were used to form levels within the analyses of variance and orthogonal polynomials. The results were also analyzed following categorization of the participants above and below the median on the Taylor Manifest Anxiety Scale. Neither of these factors yielded significant main effects or interpretable interactions involving the GSR to the control and CS words on the 44 conditioning trials. Analyses of all 45 CRs were also conducted and yielded results corresponding to the analyses of the responses induced by the control and CS words on the 44 trials.

While the analyses of the CR peaks previously described indicate a latent inhibition effect, previous analyses of GSR conditioning (Pendery \& Maltzman, 1977) suggest that these differences in CR peaks may be misleading. Examination of individual student's conditioning records in previous experiments indicated that the group data which represent averaging over participants who discover the CS-UCS contingency on different trials may provide a misleading account of what, in fact, occurred for the individual. The same may be true of the peak CR obtained by each of the three groups. We therefore determined the trial on which each participant produced their largest $\mathrm{CR}$, excluding the first trial. A maximum GSR on the first trial would be the reflection of an OR to the stimulus change represented by the first word administered Group 0. A maximum GSR to the first CS is only likely to occur in this group and not in Groups 20 and 40 which were habituated to a low level of responsivity prior to the start of the conditioning phase. The mean trial on which the largest CR occurred for students in Groups 0, 20, and 40 was $3.6(\mathrm{SD}=1.19), 9.3(\mathrm{SD}=7.77)$, and 11.5 $(\mathrm{SD}=9.76)$, respectively. The range was $2-6$ trials for Group 0, 3-45 (one participant failed to show any response) and 3-40 trials for Groups 20 and 40, respectively. Analyses by $U$ tests indicated that students in Group 0 displayed their largest CR significantly earlier than students in Group $20(\mathrm{z}=2.85)$ and Group $40(z=3.77)$. The latter two groups did not differ significantly. A test of homogeneity of variance indicated that the groups differed in this regard $\left(\mathrm{F}_{\max }=79.98\right)$. Preexposure to words therefore delayed significantly the appearance of the largest CR, excluding the response to the first CS. It also increased considerably the range of individual differences in the number of trials to attain the largest CR displayed. Turning to the mean amplitude of the largest GSR-CR displayed by the three groups, they were 2.06, 2.03, and 2.13 in Groups 0 , 20 , and 40 , respectively. There is clearly no significant difference in the average amplitude of the largest $\mathrm{CR}$ attained by the three groups.

The different CR peaks shown in Figures 1 and 2 are the consequence of the increasing range of trials over which the largest $C R$ occurred for individuals in the three groups. The apparent difference in the peak CR during conditioning induced by latent inhibition is an artifact of averaging over students displaying their largest CR over widely different ranges of trials. The largest CR obtained by the individual students is the same in the three groups when the trial on which it occurred is ignored. Similarity in amplitude of the largest CRs in the three groups is in accord with our notion of the GSR-CR as a "conditioned" voluntary OR (Pendery \& Maltzman, 1977). What remains to be accounted for is the increase in numbers of trials needed to display the largest $\mathrm{CR}$ as a consequence of preconditioning habituation.

Our interpretation is that preconditioning habituation affects the problem solving strategy of the participant. Preconditioning habituation groups require more trials, on the average, and a greater range in trials to discover the significance of the CS, because they have been given a seemingly more complex problem which included many words without the UCS. Groups 20 and 40 were misled by the preconditioning habituation phase. According to this interpretation, preconditioning habituation or preexposure of the CS serves as a masking task. But masking tasks for normal adults do not eliminate cognitive processes or problem solving activity. They simply induce different kinds from those present in the absence of masking tasks. Masking tasks usually mislead the participants, inducing false directions to their problem solving activities and thereby delaying the discovery of the significance of the CS as a signal for the UCS. Once the participants in the different groups discover the significant CS word and orient to it, they tend to orient to the same extent. They tend to show their largest GSR-OR, within the limits of overall habituation that is occurring in the 
experimental situation (Pendery \& Maltzman, 1977).

\section{GSRs to Control Words and the UCS}

Statistical analyses were conducted on the responses to $C_{1}$ and $C_{2}$ control words in order to determine whether preconditioning habituation induced by presenting many different words would have a nonspecific effect, would decrease responsivity to all words. The only significant effects obtained were due to Sex and OR level. Amount of preconditioning habituation did not yield a significant main effect, nor did it enter significantly into any interaction. Absence of a nonspecific effect is to be expected from an examination of Figure 2, which shows the responses to the $C_{2}$ words averaged over five trial blocks. It is apparent that, except for the response in Group 0 on the first trial, the three groups were quite similar.

Analyses of variance and orthogonal polynomials were also conducted on the GSR induced by the UCS on each of the 45 conditioning trials. OR level, as determined by the GSR to the first word, was a significant main effect $\left[\mathrm{F}(1,42)=20.21, \mathrm{MS}_{\mathrm{B}}=\right.$ 12.842], as was Trials $\left[\mathrm{F}(44,1848)=40.39, \mathrm{MS}_{\mathrm{W}}=\right.$ .313]. The latter effect reflects a significant decline in magnitude of the UCR over trials, a habituation effect we have seen in other experiments (Maltzman, Langdon, Pendery, \& Wolff, 1977; Maltzman, Weissbluth, \& Wolff, 1978). Preconditioning habituation did not significantly affect the UCR, nor did it enter into any interpretable interactions. Comparable results were obtained when the UCR was analyzed on the first five conditioning trials.

Thus, despite the fact that preconditioning habituation involved the single presentation of many different words, the inhibitory effect produced was specific to the CR. The GSRs induced by the control words and the UCS did not differ reliably among the three groups.

\section{Semantic Generalization}

As previously indicated, the generalization test word occurred once in each of five different subgroups within the three habituation groups. The test word, immediately preceded and followed by different control words, occurred after $5,15,25,35$, or 45 , conditioning trials. Analyses of variance and orthogonal polynomials failed to provide any evidence of differential semantic generalization following these varying numbers of conditioning trials. This failure to obtain semantic generalization with a single test trial corresponds to previous failures where the generalization test followed 9 or 10 conditioning trials (Maltzman, Gould, Barnett, Raskin, \& Wolff, 1977; Maltzman, Langdon, Pendery, \& Wolff, 1977).

\section{Extinction.}

Analyses of the GSRs induced by the CS and control words during the 10-trial extinction phase indi- cated an overall between-subject effect for OR level [Words, $\mathrm{F}(2,144)=20.86$; OR $\times$ Words, $\mathrm{F}(2,144)$ $=6.34, \mathrm{MS}_{\mathrm{W} 1}=.300$ ]. Mean GSRs for $\mathrm{C}_{1}, \mathrm{CS}$, and $C_{2}$ were $.20, .35$, and .36. Differential resistance to extinction probably occurred as suggested by the difference between $C_{1}$ and CS. Heightened responsivity to $C_{2}$ was undoubtedly a consequence of an $O R$ induced by the change from the anticipated UCS to the unanticipated $C_{2}$, as we previously reported (Maltzman, Gould, Barnett, Raskin, \& Wolff, 1977), making $\mathrm{C}_{2}$ a poor "control" for differential responsivity in extinction. The effect of preconditioning habituation was not in evidence in any obvious way as a factor influencing the rate or amount of differential extinction or overall responsivity.

Additional analyses of extinction were conducted with between-subject levels constituted by conductance level and anxiety. Conductance level yielded a significant interaction with Words. Anxiety level did not yield any significant main effects or simple interactions.

\section{Third Interval Responses}

The GSR was scored as if the UCS had been presented during extinction. Since there is no obvious within-subject control, as there is in our comparisons between control and CS words during conditioning and extinction, preconditioning habituation would be manifest here as a between-subject group effect. Two significant between-subject effects were obtained: OR $[F(1,72)=19.31]$ and Preconditioning Habituation $\left[\mathrm{F}(2,72)=3.23, \mathrm{MS}_{\mathrm{B}}=1.622\right]$. The mean GSRs obtained by Groups 0,20 , and 40 were .64, .37 , and .46, respectively. A mean GSR of .69 was obtained by the high OR students and .30 by the low OR students. It should be noted that larger mean GSRs were obtained to UCS omission than to the CS during extinction. Trials also yielded a significant effect, reflecting a progressive decline in the magnitude of the third interval response with successive evocations $\left[\mathrm{F}(9,648)=6.29, \mathrm{MS}_{\mathrm{W}}=.303\right]$.

Additional analyses of the third interval responses with levels constituted by basal conductance at the first word in the experiment for the three groups and manifest anxiety indicated that only conductance level yielded a significant effect similar to the GSR-OR. High-conductance-level participants showed a greater responsivity than low-conductance participants.

The results obtained with the third interval responses, evidence of significant latent inhibition, are rather striking. Despite the absence of any essential differences among the three groups for the last 30 conditioning trials and the absence of reliable differences in CRs during extinction, the responses following omission of the UCS reflected reliable group differences as a consequence of preconditioning habituation. These results suggest that somewhat different processes are determining the responses following UCS omission than the responses to the CS. 
But it must be noted that, in both instances, the magnitude of the initial GSR-OR was significantly related to the magnitude of the CRs, differential conditioning, and the magnitude of the responses following UCS omission.

\section{Verbalization of the Conditioning Contingency, Semantic Differential Ratings, and Stimulus Significance}

The questionnaire following the conclusion of the experiment asked the participants if they knew when the noise would occur and, if so, when. On the basis of this criterion of verbal awareness, only two participants, one in Group 0 and one in Group 40 could not verbalize the contingency between the UCS and CS.

Participants also rated the CS, generalization test word, the UCS, and selected control words on a 7point semantic differential scale with UNPLEASANT (1) and PLEASANT (7) as the polar adjectives. Participants were categorized into two groups on the basis of their semantic differential ratings of "plant," as neutral or pleasant, giving ratings of 4 and 5. Two students gave the latter rating. Four students in Group 0, three in Group 20, and seven in Group 40, rated the CS word "plant" as neutral or slightly pleasant. The remaining participants within each preconditioning habituation group rated the CS word as unpleasant. Participants within each preconditioning habituation group were randomly discarded in order to obtain a proportional number of negative and neutral raters within each preconditioning habituation group, equated for sex. This procedure yielded 19 negative raters and three neutral raters within each of the three habituation groups, all of whom verbalized the CS-UCS contingency. The two groups were categorized in this manner in order to determine whether the degree of negativity expressed on the semantic differential was related to the degree of differential GSR conditioning.

Analyses of variance and orthogonal polynomials of the GSRs induced in the last $\mathbf{4 4}$ conditioning trials yielded two relevant results. There was a significant between-subject Rating effect $\left[\mathrm{F}(1,60)=6.95, \mathrm{MS}_{\mathrm{B}}\right.$ $=11.201]$ and a significant Rating by Words interaction $\left[\mathrm{F}(2,120)=8.23, \mathrm{MS}_{\mathrm{W}}=.177\right]$. The negative raters displayed greater overall GSR responsivity as well as greater differential semantic conditioning than the neutral raters. The negative rating group obtained mean GSRs for $\mathrm{C}_{1}, \mathrm{CS}$, and $\mathrm{C}_{2}$ words of $.31, .71$, and .27 , respectively. The neutral raters obtained mean GSRs for $\mathrm{C}_{1}, \mathrm{CS}$, and $\mathrm{C}_{2}$ of $.14, .20$, and .13 , respectively.

Further analyses indicated that negative and neutral raters did not differ in the magnitude of their UCRs, responsivity to the CS during extinction, or in the magnitude of their third interval responses. Differences in the ratings of the CS word on the semantic differential were related specifically to differential
GSR conditioning and overall responsivity to the CS and control words.

It is apparent that differential semantic conditioning of the GSR may be related to the direction of semantic differential ratings among participants all of whom are verbally aware and capable of verbalizing the CS-UCS contingency. Previous semantic conditioning studies have found that only verbalizers as a group manifest differential semantic conditioning of the GSR (Maltzman, Langdon, Pendery, \& Wolff, 1977; Pendery \& Maltzman, 1977) and only verbalizers show differential semantic conditioning as manifested by semantic differential ratings. The present results indicate that students who can verbalize the CS-UCS contingency may still differ in the extent of their differential semantic conditioning of the GSR and that this difference is related to their rating of the CS word on the semantic differential.

Semantic differential ratings for the CS word "plant" and the mean of four neutral control words were examined for evidence of differential semantic conditioning and the effects of preconditioning habituation, as well as other variables. No reliable evidence of latent inhibition was found in terms of the semantic differential ratings. Differential semantic conditioning in terms of the semantic differential ratings was clearly obtained $\left[\mathrm{F}(1,72)=279.84, \mathrm{MS}_{\mathrm{W}}\right.$ $=.757]$. The mean rating for the CS word was 2.05 , while the mean rating for the control words was 4.30. OR level yielded a significant main effect $\left[\mathrm{F}(1,72)=4.96, \mathrm{MS}_{\mathrm{B}}=.705\right]$. There was also a significant $O R$ by Words interaction $[F(1,72)=$ $\left.6.05, \mathrm{MS}_{\mathrm{W}}=.757\right]$. Low-OR students obtained mean ratings of 2.36 and 4.27 for the CS and control words, respectively. High-OR students obtained mean ratings of 1.74 and 4.32 , respectively. Clearly, the magnitude of the GSR to the first word presented to the participants is significantly related to differential conditioning, as reflected by the ratings of the CS word in contrast to the ratings of the control words. Absence of a difference in the ratings of the control words by high- and low-OR students indicates that overall responsivity or some form of response bias is not the basis for their different ratings of the CS word. These results suggest that individual differences in the magnitude of the initial GSR-OR are a manifestation of something more than individual differences in peripheral responsivity (Maltzman \& Mandell, 1968). They reflect individual differences in some aspects of integrative cortical activity involved in the processing of information, which may be characterized as an aspect of attention.

Evidence of semantic generalization was also obtained in the semantic differential ratings. There was no evidence of latent inhibition in these ratings. Analysis of the ratings of "stem" and the mean control words yielded $\mathrm{F}(1,72)=40.88, \mathrm{MS}_{\mathrm{W}}=$ .405. The mean rating of the generalization test word was 3.67 in contrast to the previously indicated mean 
rating of 4.30 for the control words. Generalization subgroups receiving the test word after varying numbers of conditioning trials did not differ significantly in their ratings of the test word on the semantic differential. The UCS, of course, was rated significantly more unpleasant than the control word $\left[\mathrm{F}(1,72)=779.89, \mathrm{MS}_{\mathrm{W}}=.407\right]$. It yielded a mean semantic differential rating of 1.51 .

Presence of reliable evidence of semantic generalization on the semantic differential in the absence of evidence of generalization of the GSR calls for some explantion. Also in need of explanation is the fact that the semantic differential ratings did not vary as a function of the number of conditioning trials prior to presentation of the generalization test word. Our interpretation of the failure to obtain semantic generalization of the GSR is that a single test trial usually provides an insufficient opportunity for the students to discover the contingency between the CS and generalization test word. Repeated generalization test trials provide such an opportunity (Maltzman, Langdon, Pendery, \& Wolff, 1977) and semantic generalization is readily obtained under the latter conditions. Generalization was obtained in terms of semantic differential ratings in the present experiment despite the absence of generalization in terms of the GSR because the former was determined entirely in the semantic differential test situation. Our hypothesis is that the students discovered the contingency between the generalization test word and the CS word while completing the semantic differential ratings. In other (unpublished) studies, we have found that generalization test words absent during the conditioning phase of the experiment and presented for the first time on the semantic differential were rated in a manner showing semantic generalization. Such generalization occurred only if the students believed that the test word had been presented in the conditioning experiment.

\section{DISCUSSION}

The present experiment yielded three kinds of results which suggest the presence of a latent inhibition effect in the differential semantic conditioning of the GSR: (1) differences in the magnitude of the peak CR attained by the three groups, (2) differences in the number of trials required to attain the maximum $C R$, and (3) differences in the magnitude of the responses to UCS omission displayed by the three groups. In addition, the three preconditioning habituation groups did not differ in the amplitude of their maximum $\mathrm{CR}$ or the magnitude of their responses to the control filler words during conditioning. Likewise, they did not differ in the magnitude of their CRs during extinction or in the magnitude of their UCRs. It is important to note that the particular word used as the CS was not among those presented during preconditioning habituation. The
GSR was habituated to different words that were not associates of the CS word.

Conditioned attention, or inattention, theory cannot readily account for the above results. It implies that, since preconditioning habituation did not involve the repeated presentation of the word later employed as the CS, there was no opportunity for conditioned inattention to develop to the CS word during preconditioning habituation. As a consequence, there should be no selective, specific, inhibitory effect on the differential response to the CS word during conditioning. If conditioned inattention occurred to all words as a result of preconditioning habituation, then there should be an overall response decrement in Groups 20 and 40. Again, there is no basis for a decrease in differential responding to the CS. Likewise, it is not apparent how conditioned attention theory could account for the difference in responses to UCS omission displayed by the three preconditioning habituation groups.

Difficulties inherent in the hypothesis of conditioned attention and inattention stem in part from the fact that it is not related to a more general theory adequate to the task of accounting for human GSR conditioning. Results obtained in the present experiment can be more readily accounted for by an interpretation of the OR in classical conditioning of the GSR.

As previous analyses have indicated (Pendery \& Maltzman, 1977), the negatively accelerated growth curve of GSR semantic conditioning is a consequence of averaging over individual participants who are discovering the significant $\mathrm{CS}$ and showing their largest GSR-ORs to that stimulus on different trials. In the present experiment, examination of the largest GSR-CR obtained by the individual students regardless of the trial on which it occurred indicated that this CR did not differ significantly among the three groups. The trial on which it occurred, as well as the variability in trials, did differ among the groups. Preconditioning habituation had the effect of delaying the trial on which the student gave his or her maximum response. This, presumably, was the trial on which they discovered the significance of the $\mathrm{CS}$, that it was a signal for the UCS. Preconditioning habituation affected the rate of "conditioning" of attention, but not its amount once it occurred.

The problem of accounting for the increasing number of trials needed to attain the maximum CR as a consequence of receiving preconditioning habituation however, remains. Our hypothesis is that preconditioning habituation, and its amount, affects the problem solving strategy of the participant. It requires more trials for the participants to discover the significance of the CS following preconditioning habituation than in its absence because the students have been given a seemingly more complex problem with preconditioning habituation than without. In 
other words, preconditioning habituation serves much like a masking task. But masking tasks with normal adults do not eliminate cognitive processes or problem solving in subsequent conditioning, they simply misdirect the course of problem solving. The groups receiving preconditioning habituation required more trials than did Group 0 to discover that a particular word was related in some way to the UCS, its signal.

A basic difference between conditioned attention theory and the present OR theory is that the former assumes that the attention response is completely determined by external stimulation, salience, or relative novelty. Conditioned attention theory is based upon a conception of what we have called an involuntary or passive OR (Maltzman, 1977). The OR involved in classical conditioning and other kinds of learning, particularly at the normal human level, is generated by integrative cortical activity and is a consequence of problem solving and the discovery of the significance of relevant cues. We have called this active attention a voluntary OR. The morphology and physiology of the two kinds of OR have been discussed at some length (Homskaya, 1973; Luria, 1973; Luria \& Homskaya, 1970).

Somewhat different processes are involved in the determination of ORs that occur to the omission of the UCS, third interval responses. The third interval response is largely a conditioned involuntary OR, less under verbal regulation than the voluntary OR at the time of its elicitation. Prior instructions, sets, predispositions of various kinds, however, may affect involuntary as well as voluntary ORs (Pendery \& Maltzman, 1977).

During the course of conditioning, following the discovery of the significant CS, a neuronal model (Sokolov, 1963) is established for the stimulus situation which includes the occurrence of a CS followed by a UCS. The absence of the UCS during extinction or a test trial results in a disconfirmation of the neuronal model or anticipated stimulus sequence. A GSR-OR results. The fact that the GSRs to the omission of the UCS differed for the three groups suggests that the neuronal model or anticipations for these groups differed as a result of the presence or absence of preconditioning habituation. Since Group 0 discovered the CS-UCS contingency on an earlier trial, it had a stronger anticipation of the UCS following the CS, or a more highly developed neuronal model, than did the groups receiving preconditioning habituation. Disconfirmation of the anticipated UCS would therefore result in larger GSRORs in Group 0 than in the groups receiving preconditioning habituation. The effect of the latter treatment was to retard discovery of the significant CS and therefore provided fewer trials for the development of a neuronal model of the CS-UCS contingency.
In any case, theories that interpret latent inhibition in terms of a learned loss of stimulus salience or conditioned inattention cannot explain the obtained differences in responses to UCS omission. Conditioned attention theory is inadequate to the task if for no other reason than that it presupposes that latent inhibition is manifest only to preexposed stimuli, or stimuli similar to the preexposed stimulus.

Grings (1977) has pointed out a source of ambiguity in the theoretical interpretation of classical conditioning of the GSR in terms of the development of an OR as a result of the discovered significance of the CS. We hypothesize that a large GSR occurs to the CS because the students discover that this is a significant stimulus, significant in that it is a signal for the UCS. We know that it is a significant stimulus because it induces differentially large GSRs. There is an obvious problem of circularity present. The circle can be broken if there is available an independent measure of CS significance. In the case of classical conditioning with normal human subjects, there is such a possibility - the use of evaluative semantic differential rating scales such as the one employed in the present experiment.

Significance as defined by the semantic differential or differential GSR-ORs is used here in the sense employed by Thorndike (1931) in referring to reinforcing states of affairs, satisfiers and annoyers. Both significance and a reinforcer may be defined behaviorally in terms of approach and avoidance behavior in contrast to a neutral or nonreinforcing stimulus as suggested by Thorndike. Solarz (1960) has demonstrated that words positively evaluated on the semantic differential are related to performance indicating approach whereas negatively evaluated words are related to avoidance tendencies. An OR would be evoked by either positive or negative reinforcers, by stimuli that evoke approach or avoidance. Both are significant states of affairs.

Previous research has indicated that students who cannot verbalize the CS-UCS contingency, who have not discovered the significance of the CS, do not show differential semantic conditioning of the GSR. They likewise fail to show conditioning in terms of differential ratings on the semantic differential (Maltzman, Langdon, Pendery, \& Wolff, 1977). Results obtained in the present experiment, however, show that ratings on the semantic differential may differentiate among students who verbalize the conditioning contingency. Verbally aware students for whom the CS is negatively evaluated, i.e., significant, show greater differential conditioning of the GSR than verbally aware students for whom the CS tended to be neutral, i.e., for some reason lacked significance. These results indicate that the cognitive theorists' emphasis on the crucial role of awareness in classical conditioning (Brewer, 1974) is of limited theoretical interest. Cognitive theories of classical conditioning 
will remain barren as long as their theoretical formulations are limited, as they are now, to the positing of awareness as the causal factor in triggering the conditioning mechanism (Brewer, 1974).

Given that the significance of stimuli can be determined independently of the OR induced by the stimuli, the problem still remains as to why an OR occurs to a significant stimulus. It does so because it is an unconditioned response which occurs to such stimuli as a result of the past learning history of the individual or biological determinants.

Granting our interpretation of the role of the OR in semantic conditioning of the GSR, a problem still remains. This is to account for the great variety of results demonstrating latent inhibition in human and infrahuman organisms in a variety of different experimental situations. However, in the case of GSR conditioning, there are conflicting results (Silver, 1973; Surwit \& Poser, 1974; Zeiner, 1970). Space does not permit a detailed analysis of the latter experiments. But it should be noted that the studies with discrepant findings (Silver, 1973; Zeiner, 1974) employed a small number of preexposures and presented their results averaged over trial blocks so that it is difficult to determine their specific nature. A small number of stimulus preexposures in relation to classical conditioning with only a CS + or only one $\mathrm{CS}+$ and a CS - does not constitute a particularly effective masking task. It would not mislead normal adults in their problem solving activities to a very great extent and thereby retard discovery of the CSUCS contingency.

Lubow (1973a) has suggested that an adequate account of all of the research on latent inhibition requires both learning and attentional factors. Such factors are contained in his conception of conditioned attention. What has not been recognized is that both learning and attentional factors may also be contained in theoretical conceptions of ORs. When conditioning in normal adults is considered, problem solving also becomes a characteristic process determining ORs and latent inhibition.

It must be remembered, in conclusion, that Lubow's theory of conditioned attention is designed, primarily, to predict and explain the results of animal experiments. It is not surprising, therefore, that the theory as originally formulated would have some difficulty when extended to certain kinds of human studies. Undoubtedly, however, the present interpretation of the GSR conditioning and latent inhibition of such conditioning can be reconciled with an extension of conditioned attention theory designed more specifically to consider characteristics of human classical conditioning of the GSR.

\section{REFERENCES}

Berlyne, D. E. Conflict, arousal and curiosity. New York: McGraw-Hill, 1960.
BREWER, W. F. There is no convincing evidence of operant or classical conditioning of adult humans. In W. B. Weimer \& D. S. Palermo (Eds.), Cognition and the symbolic processes. New York: Wiley, 1974.

Carlton, P. L., \& Vogel, J. R. Habituation and conditioning. Journal of Comparative and Physiological Psychology, 1967, 63, 348-351.

DMITRIEv, A. S. On a motor technique with verbal reinforcement. Soviet Psychology and Psychiatry, 1962, 1, 8-15.

Grings, W. W. Orientation, conditioning, and learning. Psychophysiology, 1977, 14, 343-350.

HomskayA, E. D. The human frontal lobes and their role in the organization of activity. Acta Neurobiologiae Experimentalis, 1973, 33, 509-522.

Hull, C. L. Principles of behavior. New York: AppletonCentury, 1943.

Kimmel, H. D., \& BurNs, R. A. Adaptational aspects of conditioning. In W. K. Estes (Ed.), Handbook of learning and cognitive processes (Vol. 2) Conditioning and behavior theory. Hillsdale, N.J: Erlbaum, 1975.

Lubow, R. E. Latent inhibition. Psychological Bulletin, 1973, 79, 398-407. (a)

Lubow, R. E. Latent inhibition as a means of behavior prophylaxis. Psychological Reports, 1973, 32, 1247-1252. (b)

Lubow, R. E., AlEK, M., \& ARZY, J. Behavioral decrement following stimulus pre-exposure: Effects of number of preexposures, presence of a second stimulus, and interstimulus interval in children and adults. Journal of Experimental Psychology: Animal Behavior Processes, 1975, 104, 178-188.

Lubow, R. E., \& Moore, A. U. Latent inhibition: The effect of nonreinforced pre-exposure to the conditioning stimulus. Journal of Comparative and Physiological Psychology, 1959, 52, 415-419.

Lubow, R. E., Rifkin, B., \& Alek, M. The context effect: The relationship between stimulus pre-exposure and environmental pre-exposure determines subsequent learning. Journal of Experimental Psychology: Animal Behavior Processes, 1976, 2, 38-47.

Lubow, R. E., Schnur, P., \& RIFKIN, B. Latent inhibition and conditioned attention theory. Journal of Experimental Psychology: Animal Behavior Processes, 1976, 2, 163-174.

LuRIA, A. R. The working brain. New York: Basic Books, 1973.

Luria, A. R., \& Homskaya, E. D. Frontal lobes and the regulation of arousal processes. In D. I. Mostofsky (Ed.), Attention: Contemporary theory and analysis. New York: Appleton-Century-Crofts, 1970.

MacKintosh, N. J. A theory of attention: Variations in the associability of stimuli with reinforcement. Psychological Review, 1975, 82, 276-298.

Maltzman, I. Orienting in classical conditioning and generalization of the GSR to words: An overview. Journal of Experimental Psychology: General, 1977, 106, 111-119.

Maltzman, I., Gould, J., Barnett, O. J., Raskin, D. C., \& WolfF, C. Classically conditioning components of the orienting reflex to words using an innocuous and a noxious UCS under different CS-UCS intervals. Journal of Experimental Psychology: General, 1977, 106, 185-212.

Maltzman, I., \& Mandell, P. The orienting reflex as a predictor of learning and performance. Journal of Experimental Research in Personality, 1968, 3, 99-106.

Maltzman, I., Langdon, B., Pendery, M., \& Wolff, C. The GSR orienting reflex and semantic conditioning and generalization with different unconditioned stimuli. Journal of Experimental Psychology: General, 1977, 106, 141-171.

Maltzman, I., Weissbluth, S., \& WolfF, C. Habituation of orienting reflexes in repeated GSR semantic conditioning sessions. Journal of Experimental Psychology: General, 1978, 107, 309-333.

Pavlov, I. P. Conditioned reflexes. (G. S. Anrep, trans.). London: Oxford University Press, 1927.

Pendery, M., \& Maltzman, I. Instructions and the orienting reflex in "semantic conditioning" of the GSR in an innocuous 
situation. Journal of Experimental Psychology: General, 1977, 106, 120-140.

SIEGEL, S. Effects of CS habituation on eyelid conditioning. Journal of Comparative and Physiological Psychology, 1969, 68, 245-248.

SILVER, A. Effects of prior CS presentations on classical conditioning of the skin conductance response. Psychophysiology, 1973, 10, 583-588.

Sokolov, E. N. Perception and the conditioned reflex. New York: Macmillan, 1963.

Solarz, A. K. Latency of instrumental responses as a function of compatibility with the meaning of eliciting verbal signs. Journal of Experimental Psychology, 1960, 59, 239-245.

Stern, J. A. Physiological response measures during classical conditioning. In N. S. Greenfield \& R. A. Sternbach (Eds.), Handbook of psychophysiology. New York: Holt, Rinehart \& Winston, 1972.
Surwit, R. S., \& Poser, E. G. Latent inhibition in the conditioned electrodermal response. Journal of Comparative and Physiological Psychology, 1974, 86, 543-548.

Stewart, M. A., Stern, J. A., Winokur, G., \& Fredman, S. An analysis of GSR conditioning. Psychological Review, 1961, 68, 60-67.

Thorndike, E. L. Human learning. New York: Century, 1931.

Voronin, L. G., Leontiev, A. N., LuRia, A. R., Sokolov, E. N., \& VinogRadova, O. S. (Eds.), Orienting reflex and exploratory behavior. Washington, D.C: American Institute for Biological Sciences, 1965.

ZeINER, A. R. Orienting response and discrimination conditioning. Physiology and Behavior, 1970, 5, 641-646.

(Received for publication February 1, 1978; revision accepted January 9, 1979.) 